\title{
DESARROLLO DE UN EXOESQUELETO PARA EL ANTEBRAZO Y LA MUÑECA UTILIZANDO UN SOLO ACTUADOR
}

\author{
Jesús Álvarez-Pastor ${ }^{1}$, Luis Daniel Lledó ${ }^{1}$, Santiago Ezquerro ${ }^{1}$, Alicia Garrote ${ }^{2}$, Teresa Costa ${ }^{2}$, \\ Andrea Blanco ${ }^{1}$, José Vicente García-Pérez ${ }^{1}$ y Nicolás García-Aracil ${ }^{1}$ \\ ${ }^{1}$ Universidad Miguel Hernández, Av. de la Universidad, s/n, 03202, Elche. jesus.alvarezp@umh.es \\ ${ }^{2}$ Hospital la Pedrera, Dénia
}

\section{Resumen}

Las previsiones más recientes determinan que el número de accidentes cerebrovasculares aumentará en el futuro, y por este motivo, serán esenciales para acelerar la recuperación del paciente el empleo de nuevas terapias de rehabilitación asistidas por sistemas robóticos. Este artículo describe el diseño, desarrollo y control de un exoesqueleto ligero para el antebrazo y la muñeca utilizando un único actuador. Además, se ha llevado a cabo un estudio piloto con pacientes en el Hospital la Pedrera de Dénia con el objetivo de analizar la usabilidad del sistema, y los resultados se recogen en el presente estudio.

Palabras clave: robótica, rehabilitación, asistencia, exoesqueleto

\section{INTRODUCCIÓN}

Los accidentes cerebrovasculares son la principal causa de discapacidad en la población adulta europea afectando aproximadamente a 1 millón de personas anualmente, y se estima que el número absoluto de accidentes cerebrovasculares habrá aumentado un 36\% para 2025 [2]. Los supervivientes tendrán que hacer frente a un gran número de consecuencias relacionadas con trastornos cognitivos y lingüísticos, parálisis, dolor y trastornos emocionales [8].

Las terapias de rehabilitación motora tradicionales normalmente están compuestas por el paciente y su entorno físico próximo y son conducidas por el terapeuta a cargo. Aunque estas terapias son efectivas, ante el previsible aumento de accidentes cerebrovasculares en el futuro, sería necesario dotar a los terapeutas con nuevas herramientas que complementen las actuales.

Los sistemas robóticos utilizados en terapias de rehabilitación para los miembros superiores combinan interfaces y escenarios de rehabilitación orientados al robot [9]. La robótica aplicada a la rehabilitación pretende aumentar la intensidad de la terapia y la motivación del paciente [4] así como ofrecer un entorno seguro, especialmente necesario en los inicios de la rehabilitación [12]. Cabe destacar que los exoesqueletos rígidos con más de 2 grados de libertad, son, en la mayoría de los casos, pesados y voluminosos debido a los actuadores utilizados en cada articulación $[5,1$, 11]. Esto provoca que las terapias realizadas con un sistema robótico pesado sean menos cómodas que las terapias tradicionales. El desarrollo de un sistema robótico donde prime el confort del usuario afectaría positivamente al paciente y a los resultados de la rehabilitación.

El objetivo de este trabajo es el de proponer un exoesqueleto rígido para miembros superiores diseñado para llevar a cabo la rehabilitación de una forma cómoda. El exoesqueleto desarrollado es más ligero que otros sistemas robóticos similares puesto que ofrece 3 grados de libertad actuados utilizando un solo actuador. La experimentación validará si el exoesqueleto puede interactuar con un brazo humano con precisión y seguridad mientras que se realiza una terapia ocupacional simple. Además, mediremos el grado de comodidad percibido por el paciente durante la terapia con el dispositivo mediante un test de usabilidad.

\section{MÉTODOS}

\subsection{Exoesqueleto de antebrazo y muñeca}

El sistema robótico presentado en este artículo está basado en la patente registrada número WO2020183049A1 y se compone de un exoesqueleto ligero de miembro superior fijado a una mesa regulable en altura, adaptada a personas con movilidad reducida y tiene una masa de $2,25 \mathrm{~kg}$.

Se trata de un dispositivo con 3 grados de libertad (Figura 1) optimizado para terapias de rehabilitación donde el paciente tiene movilidad reducida en la muñeca y el brazo. Un ordenador se encarga del control en tiempo real mientras que la interfaz gráfica de usuario proyecta actividades sincronizadas con los movimientos 


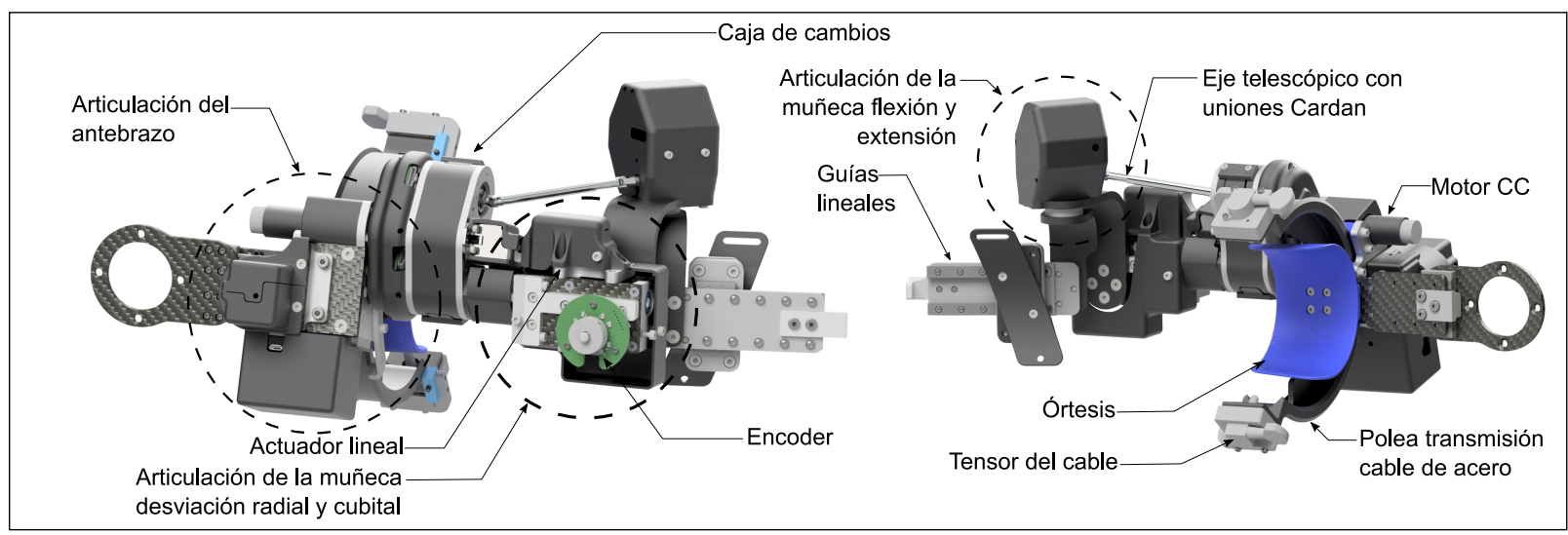

Figura 1: Exoesqueleto de antebrazo y muñeca. La carcasa de la articulación de la muñeca desviación radial y cubital ha sido eliminada para ver los componentes interiores.

del exoesqueleto. El dispositivo ha sido llamado "AWEXOS", del inglés, "arm and wrist exoskeleton".

\subsubsection{Requisitos del sistema}

Nuestro objetivo era diseñar un exoesqueleto de miembro superior para los movimientos del antebrazo y la muñeca utilizando un único actuador. De esta forma, sería posible crear un dispositivo robótico ligero que que facilitara llevar a cabo la rehabilitación de una forma más cómoda para el paciente.

El rango máximo de movimiento del antebrazo y los movimientos de la muñeca [10] están recogidos en la Tabla 1. Cabe destacar que, en las actividades de la vida diaria [7], se utiliza un rango menor. Debido a que, en algunos casos, la morfología del exoesqueleto no nos permitió alcanzar el rango máximo de la articulación, los rangos articulares del dispositivo han sido diseñados de acuerdo con los rangos típicos de la vida diaria.

Además, el actuador del exoesqueleto debe de ser capaz de actuar las articulaciones del antebrazo y la muñeca mientras que el usuario sostiene una carga de $2 \mathrm{~kg}$ en su mano. De acuerdo con [6], se establece que el par motor necesario para manipular una botella llena de 2 litros de agua es cercano a $1,4 \mathrm{~N} \cdot \mathrm{m}$.

\subsubsection{Accionamiento y transmisión}

El corazón del exoesqueleto consiste en una pequeña caja de cambios que permite el movimiento de las 3 articulaciones utilizando un pequeño actuador. Las diferentes partes de la caja de cambios están representadas en la Figura 2. Los componentes principales de la caja de cambios son: un árbol de transmisión primario y tres árboles de transmisión secundarios, cada uno de ellos acoplados a una articulación mecánica.

El árbol primario es accionado por un motor de corriente continua (MAXON DCX 19S, 17 Vátios) que incorpora una reductora con un ratio de reducción de 62:1. En el árbol primario, está montado un tren de tres engranajes, de los cuales solo un engranaje puede estar activo a la vez. Entonces, el engranaje activo transmite el par torsor a uno de los árboles secundarios permitiendo que la articulación se mueva. El ratio de reducción del conjunto es 1:1.

Como se muestra en la Figura 2, el árbol primario es hueco y cuenta con tres pares de agujeros transversales. Un eje interior, ubicado en la oquedad del árbol primario, sostiene tres pares de bolas dentro de los agujeros mencionados. El eje interior se traslada longitudinalmente a lo largo de 3 posiciones con la ayuda de un pequeño posicionador lineal (la flecha violeta en la Figura 2 muestra la traslación de eje). En el caso particular de la Figura 2, el eje interior, colocado en la posición 2, empuja el par de bolas correspondiente hacia el engranaje de en medio. De esta manera, el árbol primario es restringido radialmente con el engranaje central y permite la transmisión del par torsor hacia el árbol secundario 2. Este proceso nos permite elegir la transmisión que deseamos

Tabla 1: Rango articular del exoesqueleto y del brazo humano

\begin{tabular}{|c|c|c|c|}
\hline Movimiento articular & Brazo & Vida diaria & Exoesqueleto \\
\hline Pronación (AB) & $90^{\mathbf{O}}$ & $40^{\circ}$ & $70^{-}$ \\
\hline Supinación (AB) & $80^{\mathrm{O}}$ & $35^{\mathrm{O}}$ & $70^{\mathrm{O}}$ \\
\hline Extensión (M) & $60^{0}$ & $40^{\mathrm{O}}$ & $24^{\mathrm{O}}$ \\
\hline Flexión (M) & $90^{\underline{0}}$ & $35^{\mathrm{o}}$ & $90^{\mathrm{O}}$ \\
\hline D. Radial (M) & $20^{\underline{0}}$ & $13^{\mathrm{O}}$ & $10^{\mathrm{O}}$ \\
\hline D. Cubital (M) & $35^{\mathrm{O}}$ & $30^{0}$ & $26^{\mathrm{O}}$ \\
\hline
\end{tabular}




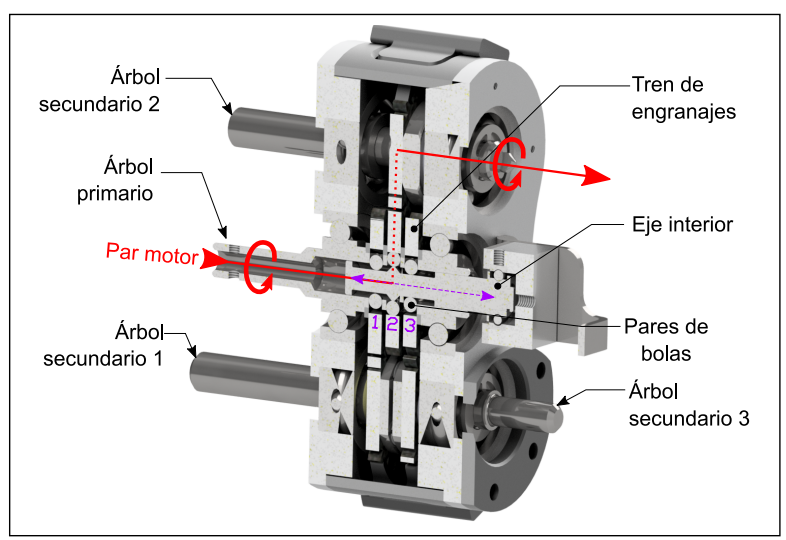

Figura 2: Caja de cambios. Vista transversal seccionada. La flecha violeta representa la traslación longitudinal del eje interior realizada por el actuador lineal. La flecha roja representa la transmisión del par motor.

actuar en cada instante.

La articulación de flexión y extensión de la muñeca está actuada por un conjunto tornillo sinfín-corona con una reducción de 10:1. Las reducciones combinadas permiten un momento torsor en la articulación de $6,82 \mathrm{~N} \cdot \mathrm{m}$. La articulación se acopla al árbol secundario 2 mediante un eje telescópico con uniones Cardan en sus extremos. La articulación de desviación radial y cubital de la muñeca tiene la misma configuración de transmisión que la articulación anterior. Un acoplamiento rígido une el árbol secundario 3 con la articulación. La articulación del antebrazo está actuada mediante cable de acero enrollado en una polea que gira sobre otra. La reducción entre poleas es de 9:1 permitiendo un par torsor en la articulación de 6,1 N.m. Con el fin de evitar que, las poleas se muevan cuando las articulaciones de la muñeca están siendo actuadas, un freno elecromagnético está acoplado al eje de la articulación del antebrazo.

Cabe destacar que los tornillos sinfín y las transmisiones por cable de acero originan incertidumbres en la posición de las articulaciones debidas a la holgura que existe entre dientes. Además, debido a la morfología de la articulación de flexión y extensión de la muñeca, la velocidad angular de las uniones Cardan no son constantes.

\subsubsection{Sensores y electrónica}

La unidad computacional central ejecuta el algoritmo de control del exoesqueleto. Un microcontrolador en tiempo real controla el posicionador lineal y su driver. Una controladora dedicada (EPOS4 COMPACT 50/5) se utiliza para controlar el motor de corriente continua
MAXON. Los dispositivos se alimentan a través de una fuente de alimentación de $150 \mathrm{~W}$ a $24 \mathrm{~V}$, $12 \mathrm{~V}$ y $5 \mathrm{~V}$ dependiendo en las necesidades.

Para solventar las incertidumbers provocadas por la holgura entre transmisiones mecánicas, las posiciones articulares son registradas a través de un encóder absoluto y rotativo (Orbis RLS) montado en cada articulación. Para solucionar los problemas en la posición de la articulación de flexión y extensión de la muñeca inducidos por la variación cinética de la unión cardan, se instaló un encóder adicional en el eje secundario 2 (Figura 2), en el que se acopla la unión Cardan.

\subsubsection{Control y software}

Se ha desarrollado un interfaz para gestionar el uso del exoesqueleto de rehabilitación AWEXOS por parte de diferentes usuarios. De esta manera se registran datos de movimiento para cada usuario. Para implementar la interfaz gráfica se ha utilizado el componente WinForms de la tecnología .NET de Windows. Winforms es una interfaz de programación incluida en .NET Framework que proporciona una manera fácil y

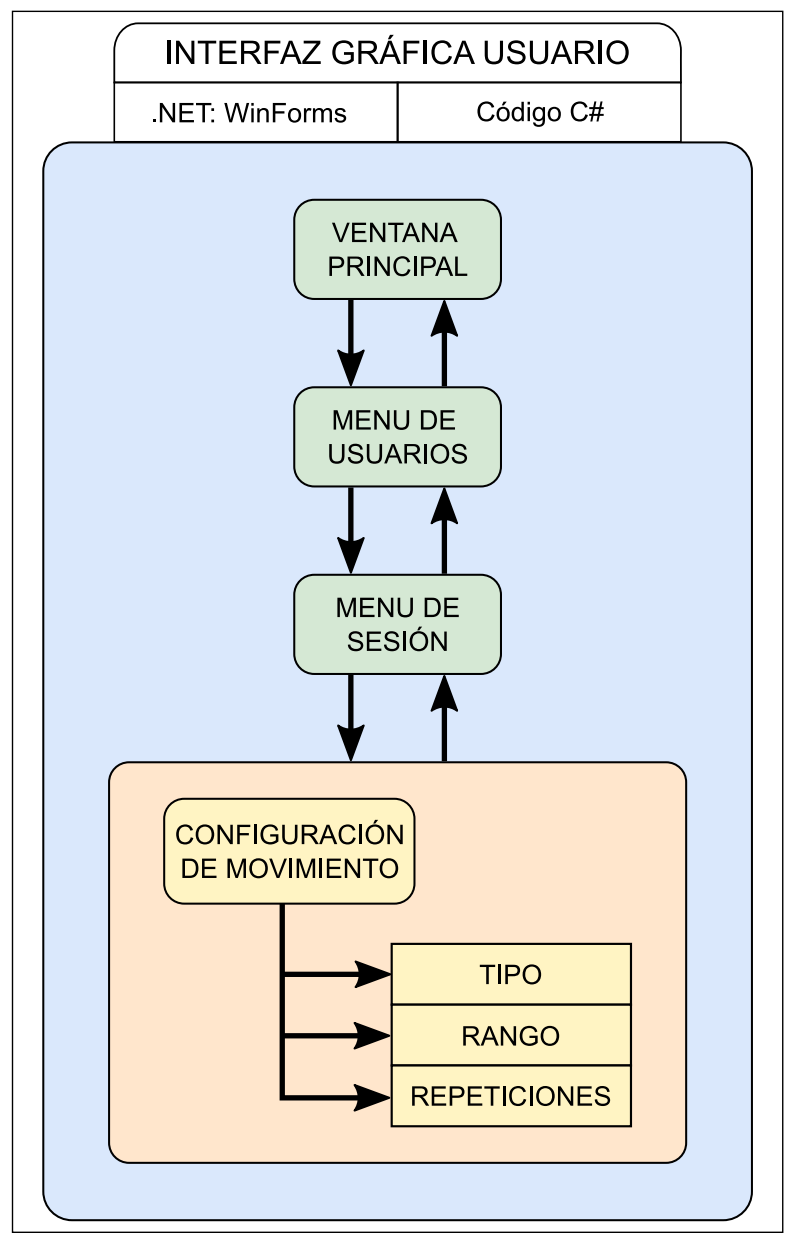

Figura 3: Interfaz gráfica de usuario desarrollada 


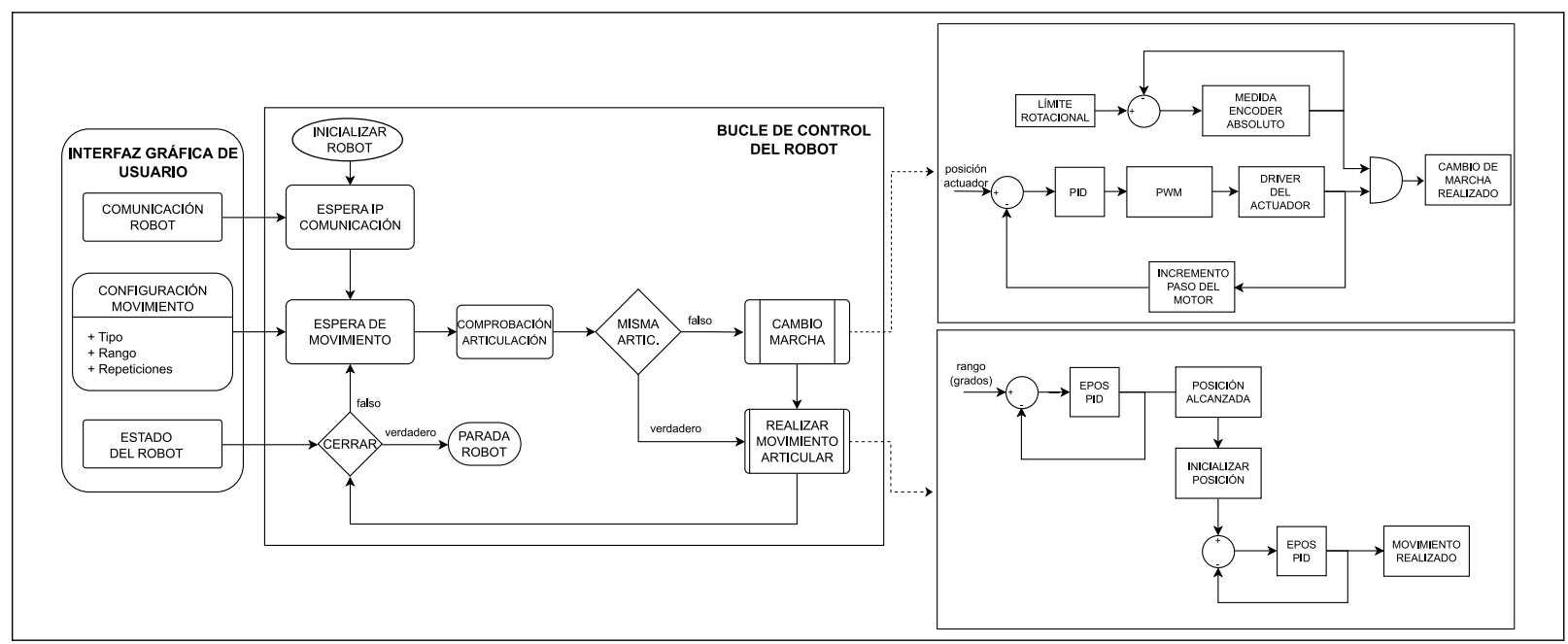

Figura 4: Diagrama de flujo del lazo de control

rápida de construir desktop apps, gestionando controles, gráficos, enlace de datos y entrada del usuario. El lenguaje de programación utilizado para el desarrollo ha sido $\mathrm{C \# .}$

En la Figura 3 se muestra un diagrama de bloques de la interfaz gráfica de usuario desarrollada. La VENTANA PRINCIPAL es el punto de entrada al sistema software y da acceso al MENÚ DE USUARIOS, el cual permite añadir, modificar o eliminar usuarios. Una vez seleccionado un usuario, se pasa a la ventana MENÚ DE SESIÓN donde se muestra los datos asociados a cada una de las sesiones de rehabilitación que ha llevado a cabo el usuario seleccionado. En el último bloque, se produce la configuración del movimiento a realizar durante la sesión. En esta parte se decide el tipo de movimiento del brazo (pronación/supinación del antebrazo, movimiento radial/cubital o flexión/extensión de la muñeca ), el rango articular y la cantidad de repeticiones que se debe realizar en la sesión.

Finalmente, el software de interfaz gráfica crea una base de datos con los datos de los usuarios con el objetivo de registrar una evolución del estado funcional del brazo del paciente a través de una serie de sesiones de rehabilitación.

El principal criterio de diseño del robot ha sido la seguridad, por lo tanto, este se ha tenido en cuenta tanto en la estructura como en el control software. Al utilizar un sólo motor para controlar tres posibles movimientos, se ha diseñado un lazo de control para dicho motor, así como para el posicionador lineal. Este lazo de control se ejecuta dentro del ordenador central. El posicionamiento del motor se lleva a cabo a través del controlador digital EPOS4 donde se le indica el número de cuentas para girar a partir de un cálculo directo del rango articular y la reducción de las articulaciones.

La figura 4 muestra el diagrama de flujo del lazo de control. En primer lugar, el robot espera la dirección IP del ordenador donde se ejecuta la interfaz gráfica de usuario. Desde esta interfaz, se envía el tipo, el rango y las repeticiones a completar, y el sistema recibe este paquete de datos con un módulo de comunicación mediante protocolo UDP (User Datagram Protocol). Estos datos son analizados para comprobar si se tiene que realizar un cambio de marcha. En la figura también se muestra el diagrama de bloques del cambio de marcha. El actuador desplaza el engranaje hasta la posición deseada y se comprueba en todo momento si el encoder absoluto varía hasta alcanzar un umbral de rotación. Mientras tanto, el motor gira paso por paso para completar el engranaje de la marcha. Una vez que el encoder absoluto registra el cambio de valor rotacional, se ha producido un cambio de marcha.

Después, se produce el giro del motor dependiendo del rango articular a partir del PID de la EPOS 4. Primero, el robot alcanza la posición deseada y luego debe volver hasta su posición inicial. $\mathrm{Al}$ completar este proceso durante un número de repeticiones, el sistema volverá a pasar a un modo de espera para recibir configuraciones de movimientos o su propia desconexión.

\subsection{Protocolo de experimentación}

Antes del inicio de la sesión, los pacientes fueron sometidos a una evaluación general para comprobar su rango de movimiento y asegurar que la experimentación se podría llevar a cabo 
con seguridad. Después de inicializar el sistema, colocamos al paciente a la izquierda del sistema robótico en una posición cómoda y atamos el exoesqueleto a su brazo. La pantalla donde se proyectaría la actividad, estaba ubicada en frente del paciente, a 1 metro de distancia del sistema robótico. La actividad consistía en un brazo humano imitando los movimientos hechos por el exoesqueleto de forma sincronizada. En la Figura 5, se muestra a un paciente utilizando el dispositivo.

Cada sesión está estructurada en tres bloques con un descanso de dos minutos por bloque. Cada bloque tiene una duración de diez minutos. La sesión se describe a continuación:

- La articulación del antebrazo realiza diez repeticiones del movimiento de pronación y otras diez repeticiones del movimiento de supinación.

- La articulación de la muñeca realiza diez repeticiones del movimiento de flexión y otras diez repeticiones del movimiento de extensión.

- La articulación de la muñeca realiza diez repeticiones del movimiento de desviación radial y otras diez repeticiones del movimiento de desviación cubital.

La evaluación del rango articular de los pacientes estableció que el rango máximo del exoesqueleto podía ser alcanzado. Después de finalizar los tres bloques de actividad, cada paciente debía responder a una corta encuesta que nos permitiría comprobar si el sistema robótico cumplía con los requisitos establecidos.

Se realizó un test de usabilidad para evaluar el grado de satisfacción del participante después de la sesión, la conformidad de acuerdo con las expectativas del paciente y el desempeño

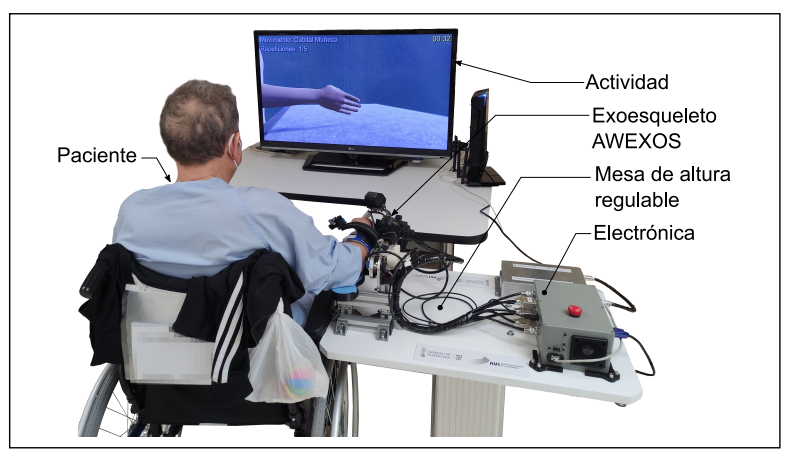

Figura 5: Paciente realizando una actividad con el dispositivo robótico del sistema robótico. Para ello, utilizamos una escala de usabilidad del sistema (SUS) [3]. Este test se componía de diez preguntas sobre juicios subjetivos de usabilidad. Las respuestas medían el nivel de acuerdo o desacuerdo de los usuarios utilizando una escala Liker (desde 1, "totalmente en desacuerdo" hasta 5, "totalmente de acuerdo") [13] con respecto al sistema robótico.

\subsection{Pacientes}

El protocolo de experimentación fue aprobado por el comité de ética del "Hospital La Pedrera". Los sujetos que participaron leyeron y comprendieron el propósito y los requisitos del estudio y firmaron un consentimiento informado antes de que la validación comenzara.

El criterio de inclusión incluía pacientes post-ictus con movilidad reducida en el antebrazo y muñeca derechos. Además, tenían que tener la capacidad de comunicarse y de comprender las instrucciones de la actividad. Finalmente, participaron en el estudio siete pacientes post-ictus, hombres y con edades comprendidas entre 40 y 70 años. En la tabla 2 se detallan los datos clínicos de los pacientes.

\section{Resultados}

La media de las puntuaciones de la encuesta realizada se muestran en la Figura 6, en la imagen de la izquierda. El gráfico naranja representa los valores de cada respuesta en la escala Liker para obtener la puntuación máxima en la escala de usabilidad del sistema. Por otro lado, el azul representa la media de las respuestas de los pacientes. Las puntuaciones basadas en la escala Liker son traducidos a un sistema de puntuación especifico determinado en [3]. Este sistema de puntuación puntúa del 0 al 100\%. Los resultados traducidos se muestran en la Figura, en la imagen de la derecha.

El valor medio de las puntuaciones obtenidas equivale a un $82 \%$ y representa la puntuación SUS del sistema robótico descrito en este trabajo.

Tabla 2: Datos de los pacientes

\begin{tabular}{|c|c|c|c|c|}
\hline ID & Índice Barthel & STREAM & Sexo & Patología \\
\hline 1 & 25 & 3 & $\mathrm{M}$ & $\begin{array}{c}\text { síndrome Guillain } \\
\text { Barré }\end{array}$ \\
\hline 2 & 40 & 7 & $\mathrm{M}$ & Ictus hemorrágico \\
\hline 3 & 30 & $* *$ & $\mathrm{M}$ & Ictus isquémico \\
\hline 4 & 65 & 0 & $\mathrm{M}$ & $\begin{array}{c}\text { Ictus isquémico } \\
\text { post-COVID-19 }\end{array}$ \\
\hline 5 & 5 & 5 & $\mathrm{M}$ & Ictus isquémico \\
\hline 7 & 45 & 14 & $\mathrm{M}$ & Post-COVID-19 \\
\hline 8 & 75 & 9 & $\mathrm{M}$ & Ictus isquémico \\
\hline
\end{tabular}




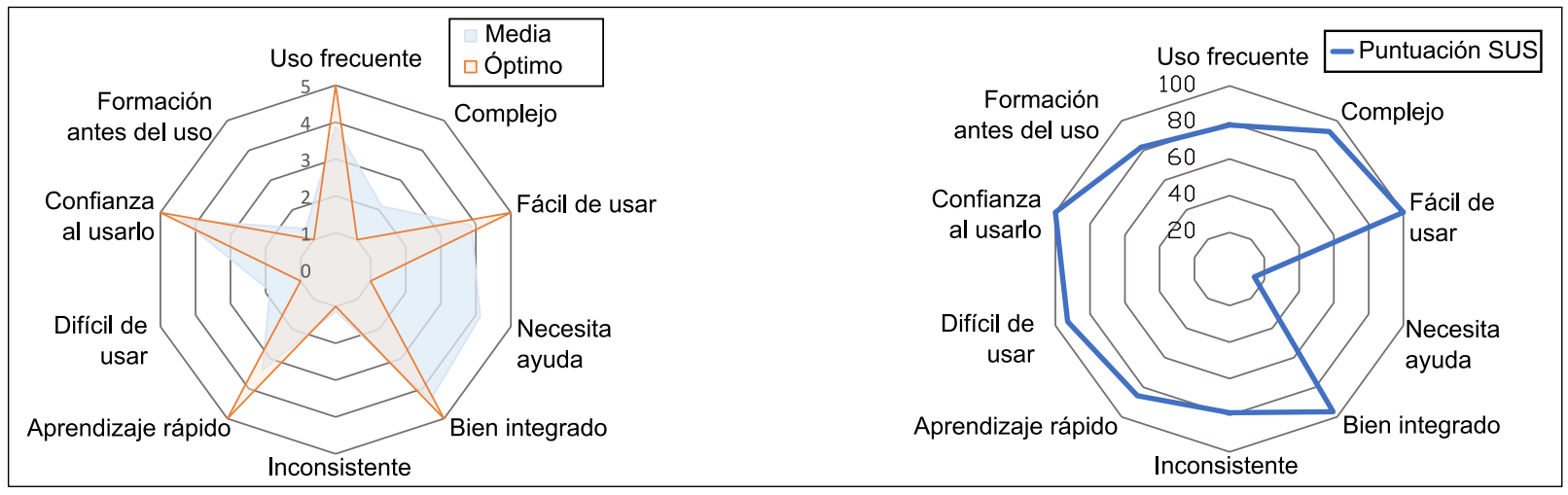

Figura 6: Respuestas a la encuesta y gráfico de puntuación SUS

Por otro lado, durante las siete sesiones, registramos el rango de movimiento alcanzado por los pacientes y todos ellos llegaron al máximo rango articular que podía abarcar el exoesqueleto.

\section{Discusión}

Un exoesqueleto de antebrazo y muñeca ha sido diseñado incorporando una novedad patentada con la cual es posible actuar tres articulaciones con un único motor de corriente continua. Esta invención aporta las siguientes ventajas para las terapias de rehabilitación y la medicina:

- Sistemas robóticos más ligeros y menos voluminosos

- Consumo de energía menor

- Sistemas robóticos más asequibles

Como hemos visto en la Figura 6, la media de las respuestas es cercana a las respuestas óptimas, en la mayoría de los casos. Cabría destacar que en la respuesta que evaluaba la necesidad de ayuda para utilizarlo difiera de la respuesta óptima debido a que se probó en pacientes con movilidad reducida tras un accidente cerebrovascular. Por lo tanto, necesitaban de ayuda para instalar el sistema robótico en su brazo.

La puntuación SUS calculada es igual a un $82 \%$ lo que implica que la usabilidad del sistema está comprendida entre "BUENA" y "EXCELENTE", demostrando que el dispositivo es confortable en su uso. Experimentaciones adicionales podrían probar el impacto positivo de estas terapias utilizando exoesqueletos ligeros.

\section{Conclusión}

El exoesqueleto con tres grados de libertad y un actuador ha sido validado para ser empleado en terapias de rehabilitación en pacientes post-ictus. La usabilidad del sistema es buena de acuerdo con la escala SUS y ofrece varias ventajas. Se realizarán futuras experimentaciones para validar las posibles ventajas que tiene de cara a la rehabilitación de los pacientes.

\section{Agradecimientos}

Gracias a Gemma Mas y María Victoria Pomares del Hospital de la Pedrera. Este estudio ha sido subvencionado por la Agència Valenciana de la Innovació (INNVAL10/19/065), el Ministerio de Ciencia, Innovación y Universidades, perteneciente a la Agencia Estatal de Investigación (AEI), a través de la ayuda PEJ2018-002670-A y de la ayuda PEJ2018-002684-A. (Cofinanciado por el Fondo Social Europeo. FSE invierte en tu futuro), y por la Consellería de Educación, Cultura y Deporte de la Generalitat Valenciana (GVA), a través de la ayuda APOTIP/2020/014.

\section{English summary}

DEVELOPMENT OF AN ACTIVE EXOSKELETON FOR FOREARM AND WRIST REHABILITATION USING A SINGLE ACTUATOR
Abstract
Recent research states that the number of strokes will increase in the future. For this reason, it is paramount to introduce new rehabilitation therapies to accelerate the recovery of the patient. This article describes the design, development and control of a light exoskeleton for the forearm and wrist using a single actuator. Furthermore, a pilot study has been done with patients in La Pedrera Hospital in 
Denia to analyse the system's usability. The results are presented in this study.

Keywords: robotics, rehabilitation, assistance, exoskeleton.

\section{Referencias}

[1] Badesa, F. J., Llinares, A., Morales, R., Garcia-Aracil, N., Sabater, J. M., \& Perez-Vidal, C. (2014). Pneumatic planar rehabilitation robot for post-stroke patients. Biomedical Engineering: Applications, Basis and Communications, 26(02), 1450025.

[2] Béjot, Y., Bailly, H., Durier, J., \& Giroud, M. (2016). Epidemiology of stroke in Europe and trends for the 21st century. La Presse Médicale, 45(12), e391-e398.

[3] Brooke, J. (1996). SUS-A quick and dirty usability scale. Usability evaluation in industry, 189(194), 4-7.

[4] Catalán, J. M., García-Pérez, J. V., Blanco, A., Martínez, D., Lledó, L. D., \& García-Aracil, N. (2021). Differences in Physiological Reactions Due to a Competitive Rehabilitation Game Modality. Sensors, 21(11), 3681.

[5] Culmer, P. R., Jackson, A. E., Makower, S., Richardson, R., Cozens, J. A., Levesley, M. C., \& Bhakta, B. B. (2009). A control strategy for upper limb robotic rehabilitation with a dual robot system. IEEE/ASME Transactions on Mechatronics, 15(4), 575-585.

[6] Díez, J. A., Blanco, A., Catalán, J. M., Badesa, F. J., Sabater, J. M., \& Garcia-Aracil, N. (2017). Design of a prono-supination mechanism for activities of daily living. In Converging Clinical and Engineering Research on Neurorehabilitation II (pp. 531-535). Springer, Cham.
[7] Gates, D. H., Walters, L. S., Cowley, J., Wilken, J. M., \& Resnik, L. (2016). Range of motion requirements for upper-limb activities of daily living. American Journal of Occupational Therapy, 70(1), 7001350010p1-7001350010p10.

[8] Lai, S. M., Studenski, S., Duncan, P. W., \& Perera, S. (2002). Persisting consequences of stroke measured by the Stroke Impact Scale. Stroke, 33(7), 1840-1844.

[9] Loureiro, R. C., Harwin, W. S., Nagai, K., \& Johnson, M. (2011). Advances in upper limb stroke rehabilitation: a technology push. Medical \& biological engineering \& computing, 49(10), 1103-1118.

[10] Norkin, C. C., \& White, D. J. (2016). Measurement of joint motion: a guide to goniometry. FA Davis.

[11] Rehmat, N., Zuo, J., Meng, W., Liu, Q., Xie, S. Q., \& Liang, H. (2018). Upper limb rehabilitation using robotic exoskeleton systems: a systematic review. International Journal of Intelligent Robotics and Applications, 2(3), 283-295.

[12] Sale, P., Franceschini, M., Mazzoleni, S., Palma, E., Agosti, M., \& Posteraro, F. (2014). Effects of upper limb robot-assisted therapy on motor recovery in subacute stroke patients. Journal of neuroengineering and rehabilitation, 11(1), 1-8.

[13] Likert, R. (1974). A method of constructing an attitude scale. Scaling: A sourcebook for behavioral scientists, 233-243.

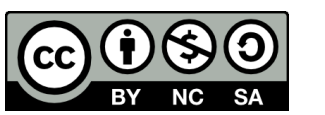
(C) 2021 by the authors. Submitted for possible open access publication under the terms and conditions of the Creative Commons Attribution CC BY-NC-SA 4.0 license (https://creativecommons.org/licenses/by-nc-sa/4.0/deed.es). 